\title{
Computed tomographic coronary angiography in patients with surgically treated type $A$ aortic dissection: preliminary results
}

\author{
Andrea B. Rosskopf • Rahel Bugmann • \\ Volkhard Goeber • Daniel Ott • Otto Hess • \\ Thierry Carrel $\cdot$ Hanno Hoppe
}

Received: 10 June 2009 / Accepted: 1 July 2009 /Published online: 4 September 2009

(C) Am Soc Emergency Radiol 2009

\begin{abstract}
Acute type A aortic dissection is a serious emergency with a mortality rate of up to $40 \%$ within the first $24 \mathrm{~h}$ when left untreated. Surgical therapy needs to be initiated promptly. Due to this urgent situation, preoperative evaluation of the coronary arteries is not routinely performed in these patients. The aim of this study was to evaluate the accuracy of 64-slice computed tomography angiography (CTA) for postoperative coronary artery assessment in these patients. Ten consecutive patients with two or more cardiovascular risk factors were prospectively enrolled. Patients had type A aortic dissection treated surgically with a supracoronary graft of the ascending aorta. Performance of CTA to exclude significant stenosis ( $>50 \%$ lumen narrowing) and/or coronary artery dissection was compared with quantitative coronary angiography. A total of 147 segments were evaluated. Three segments (2\%) were excluded from analysis. CTA correctly assessed one of three significant stenoses in three patients and correctly excluded coronary artery disease (CAD) in six of ten patients. One patient was rated false positive. Overall accuracy, sensitivity, specificity, positive predictive value
\end{abstract}

\footnotetext{
A. B. Rosskopf $\cdot$ R. Bugmann · D. Ott $\cdot$ H. Hoppe

Radiology, Bern University Hospital,

Bern, Switzerland

V. Goeber $(\bowtie) \cdot T$. Carrel

Department of Cardiac Surgery,

Bern University Hospital (Inselspital),

Freiburgstrasse,

3010 Bern, Switzerland

e-mail: volkhard.goeber@insel.ch

O. Hess

Cardiology, Bern University Hospital,

Bern, Switzerland
}

(PPV), and negative predictive value (NPV) of CT for identifying coronary artery disease by segment was $98 \%$, $33 \%, 99 \%, 50 \%$, and $99 \%$, respectively $(P<0.05)$. By patient, it was $70 \%, 33 \%, 86 \%, 50 \%$, and $75 \%$, respectively. No coronary artery dissection was found. Noninvasive CTA may be a viable alternative to conventional angiography for postoperative coronary artery evaluation in patients with surgically treated type A aortic dissection and cardiovascular risk factors. An NPV of $99 \%$ should allow for reliable exclusion of CAD. Further studies with higher patient numbers are warranted.

Keywords Aortic dissection · Cardiac CT · CTA

\section{Introduction}

Acute type A aortic dissection is a life-threatening condition with a mortality rate of up to $40 \%$ within the first $24 \mathrm{~h}$ when left untreated [1]. Surgical therapy is the treatment of choice. In this situation, preoperative evaluation of the coronary arteries is not routinely recommended in these patients [2]. Due to common risk factors, these patients may be at high risk for coronary artery disease.

Conventional coronary angiography is traditionally the gold standard for evaluation of patients with suspected coronary artery disease [3]. The introduction of multidetector-row computed tomography (CT) scanners paved the way to use this modality for evaluation of coronary artery disease. Recently, 64-slice CT angiography (CTA) with high temporal and spatial resolution was introduced for identification of stenotic and nonstenotic coronary artery plaques [4]. Several studies have demonstrated that CTA detects significant $(>50 \%)$ coronary stenosis with excellent accuracy [3-6]. 
Especially, the high negative predictive value of CTA supposedly allows for reliable exclusion of significant coronary artery disease.

The purpose of this study was to evaluate the accuracy of 64-slice CTA for postoperative coronary artery assessment in patients with type A aortic dissection and supracoronary graft replacement of the ascending aorta in correlation with conventional coronary angiography.

\section{Materials and methods}

\section{Study population}

Ten consecutive patients with two or more cardiovascular risk factors (nicotine abuse, dyslipoproteinemia, diabetes mellitus, arterial hypertension, obesity, and positive family history) were prospectively enrolled (four women and six men). Mean age was $67 \pm 7.2$ years. All patients had aortic type A aortic dissection treated with supracoronary graft replacement of the ascending aorta. Patients first underwent CTA of coronary arteries with retrospective electrocardiogram (ECG)-gating for screening of significant coronary artery disease and second conventional angiography for confirmation. Both CTA and conventional coronary angiography were performed after surgery within a 6-month interval. The time difference between both modalities was no longer than 4 weeks. Exclusion criteria for CTA were previous coronary stent placement, bypass graft surgery, presence of tachyarrhythmia, atrial fibrillation, renal insufficiency (serum creatinine $>120 \mu \mathrm{mol} / \mathrm{L}$ ), and known allergy to contrast media. This HIPPA-compliant retrospective study was approved by the local ethics committee and informed consent was waived.

\section{CT angiography}

Image acquisition was performed using a 64-slice CT scanner (Somatom Sensation 64 Cardiac; Siemens, Forchheim, Germany). Scanning was performed in a craniocaudal direction. In the absence of contraindications, patients with a resting heart rate of more than 70 beats per minute received $50-100 \mathrm{mg}$ metoprolol per os $1 \mathrm{~h}$ prior to CT scanning. At the beginning of the procedure, blood pressure and heart rate were checked. ECG signal was monitored throughout the scan, and all patients were in sinus rhythm. The examination protocol used a tube voltage of $120 \mathrm{kV}$ and an effective tube current-time product of $750 \mathrm{mAs}_{\text {eff }}$ with acquisition of 64 slices per gantry rotation using Z-sharp technology with a collimation of $64 \times 0.6 \mathrm{~mm}$ and a gantry rotation time of $330 \mathrm{~ms}$ and a table feed of $3.8 \mathrm{~mm}$ per rotation resulting in a pitch of 0.2 [7]. No ECGdependent dose modulation was applied.
Contrast material was administered via the left cubital vein. Bolus tracking technique was used to determine scan delay. When a threshold of 100 Hounsfield units was reached at the level of the coronary arteries in the ascending aorta, a delay of $8 \mathrm{~s}$ was applied prior to scanning. A contrast injection protocol with injection of $90 \mathrm{~mL}$ of nonionic contrast material (Ultravist 370; Schering, Berlin, Germany) with a flow rate of $4 \mathrm{~mL} / \mathrm{s}$ followed by a saline chaser bolus of $50 \mathrm{~mL}$ with the same flow rate was used.

Image analysis

Axial images were reconstructed from raw data with an effective slice thickness of $0.75 \mathrm{~mm}$ and an increment of $0.5 \mathrm{~mm}$ at $60 \%$ of RR-interval. In case of motion artifacts, further reconstructions had to be done at different time points within the cardiac cycle. We used an individually adapted field of view, a $512 \times 512$ matrix and a mediumsmooth convolution kernel (B25) resulting in a spatial resolution of $0.4 \mathrm{~mm}^{3}$.

CT images were analyzed by a board-certified radiologist specialized in cardiovascular imaging and a last year radiology resident with special interest in cardiovascular imaging as consensus reading. Readers were blinded to findings of conventional coronary angiography using a combination of axial images, multiplanar (MPR) views including centerline curved MPRs of the coronary arteries on an interactive viewing workstation (Leonardo, Siemens). Analysis was based on 15 segments established by American Heart Association (AHA) criteria comprising the right coronary artery (four segments), left main stem [1], left anterior descending artery and branches [5], and circumflex artery and branches [5, 8]. Each segment was classified as stenosed ( $\geq 50 \%$ lumen narrowing) by visual estimation. For each segment, presence of dissection was evaluated. Segments were excluded from analysis if absent, heavily calcified, not opacified due to proximal occlusion, or poor image quality.

\section{Conventional coronary angiography}

Conventional coronary angiography was performed via a femoral approach applying approximately $40 \mathrm{~mL}$ of nonionic contrast material. A minimum of six orthogonal views were obtained. All patients underwent CTA and conventional angiography within 4 weeks. Conventional angiographic images were evaluated by a board-certified cardiologist blinded to CTA findings. Assessment of diameter stenosis was by visual estimation with quantification of lumen narrowing. The AHA classification of 15 coronary segments and stenosis grading was used for both CTA and coronary angiography. 
Table 1 Analysis of significant $(>50 \%)$ coronary artery stenosis in 147 vessel segments of ten patients. Three segments $(2 \%)$ had to be excluded from the analysis

\begin{tabular}{llllr}
\hline & TP & FP & FN & TN \\
\hline Per segment & 1 & 1 & 2 & 143 \\
LM & 0 & 0 & 0 & 8 \\
LAD & 1 & 1 & 0 & 38 \\
LCX & 0 & 0 & 2 & 20 \\
RCA & 0 & 0 & 0 & 47 \\
Per patient & 1 & 1 & 2 & 6 \\
\hline
\end{tabular}

$L M$ left main coronary artery, $L A D$ left anterior descending artery, $L C X$ circumflex artery, $R C A$ right coronary artery, $T P$ true positive, $F P$ false positive, $T N$ true negative, $F N$ false negative

Statistical analysis

Statistical analysis was performed with GraphPad statistical software (Prism v. 4.01 and InStat v 3.0; GraphPad Software, San Diego, CA, USA). Continuous data were expressed as mean with standard deviation. Measures of diagnostic accuracy of CTA for coronary artery stenosis including sensitivity, specificity, accuracy, negative predictive value, and positive predictive value, were calculated on a per segment and per patient basis.

\section{Results}

In all patients, CTA was performed without complications. Of a total of 150 segments, 147 segments were evaluated. Three of 150 segments (2\%) had to be excluded from analysis due to heavy calcification $(n=2)$ and nonopacification of segment due to proximal vessel occlusion $(n=1)$. No coronary artery dissection was found.

Patient-based analysis

Patient-based analysis is summarized in Table 1. Of ten patients, three patients $(30 \%)$ had significant $(>50 \%)$ stenosis in at least one coronary artery segment. Thereof, one was correctly identified by CTA and two were missed. In these three patients with one significant stenosis each, interventional treatment with angioplasty and/or stent was performed in two patients who presented with ischemic sequelae. One patient had a significant stenosis without ischemic sequelae and did not undergo interventional treatment. None of these patients was treated with surgical bypass. One patient was classified as with significant stenosis by CTA, but demonstrated only minor stenosis on conventional coronary angiography and was false positive. In another six patients, significant coronary artery stenosis was correctly ruled out by CTA. The findings result in a patient-based accuracy of $70 \%$ and a confidence interval (CI) of $54-86 \%$, a sensitivity of $33 \%$ (CI 7-60\%), specificity of $86 \%$ (CI $74-97 \%$ ), a positive predictive value of $50 \%$ (CI $10-90 \%$ ), and a negative predictive value of $75 \%$ (CI 65-85\%; Table 2).

Segment-based analysis

In a total of 147 segments, three significant stenoses $(>50 \%)$ were diagnosed at conventional coronary angiography (Table 1). The distribution of disease concerning the coronary arteries was left anterior descending artery $(n=1)$ and circumflex artery $(n=2)$. No significant stenoses were found within the right coronary artery. Of these stenoses, one was correctly detected by CTA and two were false negative (Fig. 1). In 143 segments, significant coronary artery stenosis was correctly ruled out by CTA. The segment-based analysis demonstrated an accuracy of $98 \%$ (CI, 97-99\%), a sensitivity of 33\% (CI, 1-60\%), a specificity of $99 \%$ (CI, 99-100\%), a positive predictive value of $50 \%$ (CI, 10-90\%), and a negative predictive value of 99\% (CI, 98-99\%; Table 2). These findings were statistically significant $(P<0.05)$.

\section{Discussion}

Acute A dissection of the thoracic aorta is a life-threatening emergency condition. It requires a timely and accurate diagnosis to initiate successful treatment. Without surgical treatment, the mortality rate may be as high as $40 \%$ on day one and $70 \%$ within the first week [1]. This patient group is also of high risk for other diseases such as coronary artery disease. In a previous postmortem study, 161 cases of aortic dissection were analyzed and $25 \%$ of cases demonstrated evidence of severe coronary atherosclerosis [9]. These findings may have important implications on the diagnostic

Table 2 Results of significant ( $>50 \%$ ) coronary artery stenosis analysis per segment in 147 vessel segments of ten patients and per patient

\begin{tabular}{llllllllllll}
\hline & Sensitivity & CI & Specificity & CI & Accuracy & CI & PPV & CI & NPV & CI & $P$ value \\
\hline Per segment & $33 \%$ & $1-60$ & $99 \%$ & $99-100$ & $98 \%$ & $97-99$ & $50 \%$ & $10-90$ & $99 \%$ & $98-99$ & 0.04 \\
Per patient & $33 \%$ & $7-60$ & $86 \%$ & $74-97$ & $70 \%$ & $54-86$ & $50 \%$ & $10-90$ & $75 \%$ & $65-85$ & 0.5 \\
\hline
\end{tabular}

$P P V$ positive predictive value, $N P V$ negative predictive value, $C I$ confidence interval 
Fig. 1 A 67-year-old patient's postsurgical treatment of type A aortic dissection. a Volumerendered image of CT coronary angiography (CTA) dataset demonstrating calcification of the circumflex artery and an adjacent stenosis (arrow). b Magnified volume-rendered image of CTA demonstrates calcification of the circumflex artery and marginal artery and adjacent circumflex artery stenosis (arrow). c Curved 2D reformat image of circumflex artery CTA demonstrates hyperdense arterial calcifications and a stenosis of the circumflex artery (arrow), which was graded nonsignificant. $\mathbf{d}$ Conventional coronary angiography also demonstrates circumflex artery stenosis, which was graded to be significant $(>50 \%)$
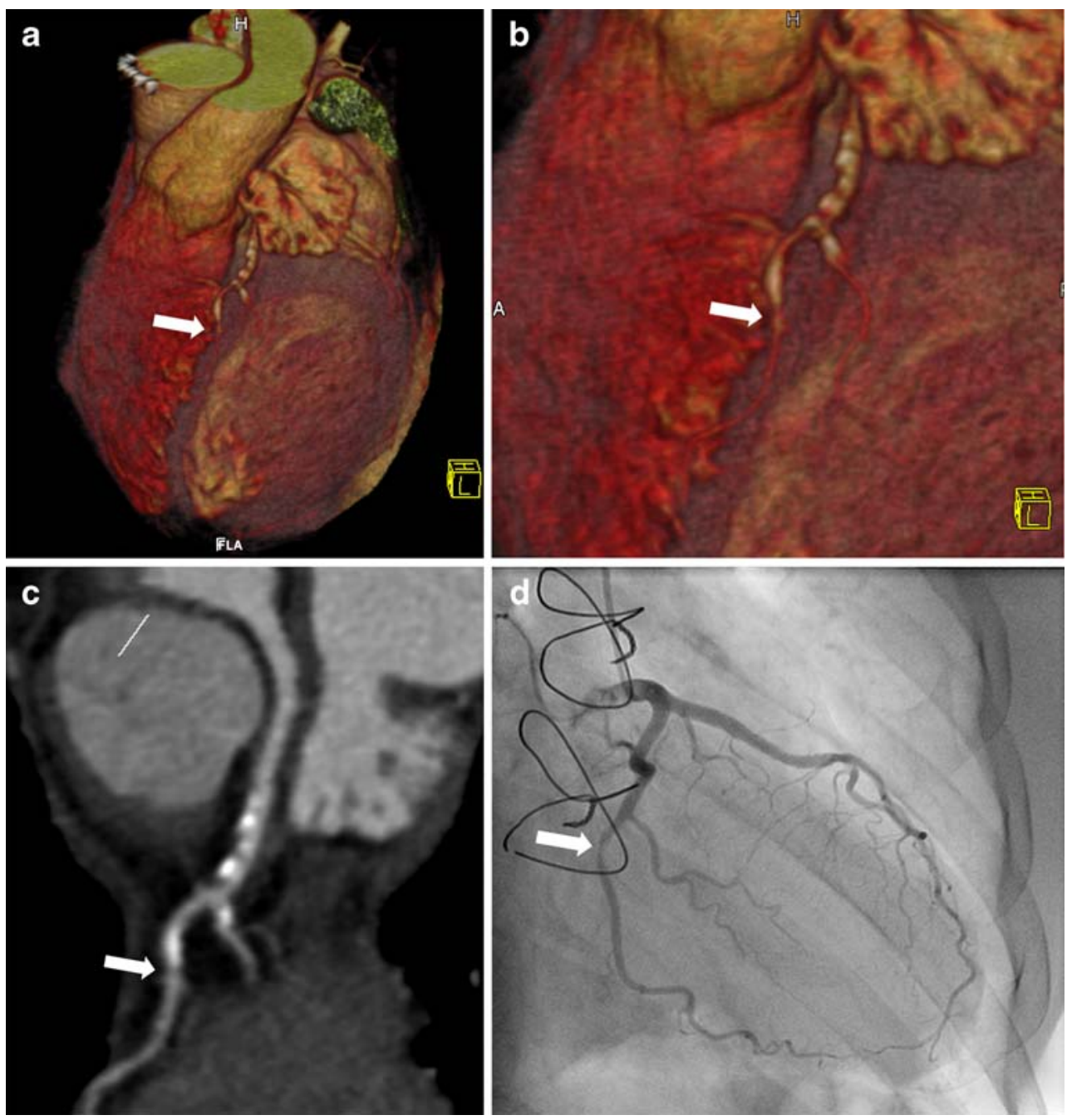

modality and surgical treatment of acute aortic dissection, particularly in an elderly patient population. Perioperative transesophageal echocardiography is appropriate to visualize the coronary ostia including the proximal portion of the coronary vessels and is practical to exclude extension of aortic dissection into the coronary vessels [10]. Accordingly, other imaging modalities are required to diagnose atherosclerosis, which may be located more distally. Conventional coronary angiography, which is the gold standard for coronary artery visualization, is associated with certain risks. Advancement of a diagnostic catheter into the false lumen may potentially extend dissection [11]. In case of present coronary artery dissection, conventional coronary angiography may not be possible from the true lumen. Furthermore, there may be substantial delay of surgical treatment caused by preceding coronary angiography.

In the literature, reports on whether to use coronary angiography prior to surgical repair of acute type A aortic dissection or not and its effect on postoperative mortality remain controversial [12-14]. To date, American College of Cardiology guidelines do not contain a clear consensus concerning preoperative coronary angiography in patients with acute aortic dissection [15]. A study by Motallebzadeh et al. analyzed the value of coronary angiography regarding concomitant coronary artery bypass graft, surgery delay, morbidity, and mortality in patients with acute type A aortic dissection [2]. Their study demonstrated that coronary angiography did not affect the occurrence of coronary artery bypass graft and was not associated with improved hospital survival. The authors concluded that coronary angiography caused a considerable delay to surgery and do not routinely recommend preoperative conventional coronary angiography in these patients.

In our institution, coronary angiography is not routinely performed prior to surgery in patients with acute type A aortic dissection. Although there may be common risk factors for both dissection and coronary artery disease, pathophysiology of each disease is fundamentally different. Routine use of conventional coronary angiography prior to surgery seems not to be supported due to a relatively low incidence $(11 \%)$ of coronary artery disease in these patients [16]. However, in our study, $30 \%$ (three of ten) of patients 
had evidence of coronary artery disease and a postoperative rule-out of coexistent coronary artery disease seemed quite reasonable.

State of the art multislice-CT scanners allow for noninvasive high definition coronary angiography. In the vast majority of our patients, this technique allowed for evaluation of all 15 coronary tree segments for significant coronary artery stenosis. No more than three out of 147 (2\%) segments had to be excluded from analysis due to heavy calcification or nonopacification of segment due to proximal vessel occlusion. This number is somewhat lower than previous limits for a 64 -slice CT scanner [3, 5, 17]. In the present study, particularly the negative predictive values were high ( $99 \%$ per segment and $75 \%$ per patient), which is supported by findings of previous studies and underline the usefulness of CTA to exclude coronary artery disease $[3,5]$. Despite acceptable accuracies for stenosis detection ( $98 \%$ per patient and $70 \%$ per segment), sensitivities (33\% for both per patient and per segment) were low in comparison to metaanalysis data reporting sensitivities ranging from $73 \%$ to $99 \%[18,19]$. Sensitivities were low mainly due to the fact that the number of patients was also low and that these are preliminary results. Due to the limited number of patients in our study, these findings should not be overestimated.

Contrast-enhanced multidetector-row $\mathrm{CT}$ is a fast imaging modality which allows for detection of both aortic and/or coronary artery dissection (1-2\% of cases) and coronary artery disease at the same time. Since contrast-enhanced CTA is performed in the emergency room in patients with acute chest pain and suspected aortic dissection, it has already replaced previous invasive diagnostic procedures and currently represents the imaging modality of choice [20]. CT can image both vessel lumen and wall, saves valuable time regarding preoperative work-up, and additional coronary artery bypass graft surgery can be performed if necessary. A recently described CT protocol for the so-called "triple rule-out" uses a dedicated contrast media regimen and is ECG-gated allowing simultaneous visualization of the pulmonary arteries, thoracic aorta, and coronary arteries within a single scan [21]. Especially, the use of modern dual-source $\mathrm{CT}$ enables rapid scanning, high image quality, and dose reduction [22]. This modern technique may not only prove particularly useful in patients with type A aortic dissection prior to surgery, but also for postoperative follow-up studies.

A major limitation of this study is the preliminary situation with a limited number of patients enrolled. Accordingly, adverse events that occur at a low rate could have been missed. Furthermore, coronary artery CTA was performed postoperatively and not in combination with a preoperative CTA of the thoracic aorta. Further studies using preoperative CTA for evaluation of both artery dissection and coronary artery disease appear reasonable.
In conclusion, preliminary results of noninvasive coronary $\mathrm{CT}$ angiography for postoperative evaluation of the coronary arteries in patients after surgical repair of type A aortic dissection were ambiguous. Despite a high negative predictive value of $99 \%$, which may allow for exclusion of coronary artery disease, sensitivities were lower than previously reported, which may be mainly attributable to limited patient numbers. Further studies with higher patient numbers are warranted.

\section{References}

1. Anagnostopoulos CE, Prabhakar MJ, Kittle CF (1972) Aortic dissections and dissecting aneurysms. Am J Cardiol 30:263-273

2. Motallebzadeh R, Batas D, Valencia O, Chandrasekaran V, Smith J, Brecker $S$ et al (2004) The role of coronary angiography in acute type A aortic dissection. Eur J Cardiothorac Surg 25:231235

3. Muhlenbruch G, Seyfarth T, Soo CS, Pregalathan N, Mahnken AH (2007) Diagnostic value of 64-slice multi-detector row cardiac CTA in symptomatic patients. Eur Radiol 17:603-609

4. Leber AW, Knez A, von Ziegler F, Becker A, Nikolaou K, Paul S et al (2005) Quantification of obstructive and nonobstructive coronary lesions by 64-slice computed tomography: a comparative study with quantitative coronary angiography and intravascular ultrasound. J Am Coll Cardiol 46:147-154

5. Ghostine S, Caussin C, Habis M, Habib Y, Clement C, SigalCinqualbre A et al (2008) Non-invasive diagnosis of ischaemic heart failure using 64-slice computed tomography. Eur Heart J. 29:2133-2140

6. Mowatt G, Cook JA, Hillis GS, Walker S, Fraser C, Jia X et al (2008) 64-Slice computed tomography angiography in the diagnosis and assessment of coronary artery disease: systematic review and meta-analysis. Heart 94:1386-1393

7. Flohr TG, Stierstorfer K, Ulzheimer S, Bruder H, Primak AN, McCollough $\mathrm{CH}$ (2005) Image reconstruction and image quality evaluation for a 64-slice CT scanner with z-flying focal spot. Med Phys 32:2536-2547

8. Austen WG, Edwards JE, Frye RL, Gensini GG, Gott VL, Griffith LS et al (1975) A reporting system on patients evaluated for coronary artery disease. Report of the Ad Hoc Committee for Grading of Coronary Artery Disease, Council on Cardiovascular Surgery, American Heart Association. Circulation 51(4 Suppl):5-40

9. Larson EW, Edwards WD (1984) Risk factors for aortic dissection: a necropsy study of 161 cases. Am J Cardiol 53:849-855

10. Ballal RS, Nanda NC, Gatewood R, D'Arcy B, Samdarshi TE, Holman WL et al (1991) Usefulness of transesophageal echocardiography in assessment of aortic dissection. Circulation 84:19031914

11. Hart WL, Berman EJ, La CR (1963) Hazard of retrograde aortography in dissecting aneurysm. Circulation 27:1140-1142

12. Kern MJ, Serota H, Callicoat P, Deligonul U, Lee WH, Aguirre F et al (1990) Use of coronary arteriography in the preoperative management of patients undergoing urgent repair of the thoracic aorta. Am Heart J 119:143-148

13. Penn MS, Smedira N, Lytle B, Brener SJ (2000) Does coronary angiography before emergency aortic surgery affect in-hospital mortality? J Am Coll Cardiol 35:889-894

14. Creswell LL, Kouchoukos NT, Cox JL, Rosenbloom M (1995) Coronary artery disease in patients with type A aortic dissection. Ann Thorac Surg 59:585-590 
15. Scanlon PJ, Faxon DP, Audet AM, Carabello B, Dehmer GJ, Eagle KA et al (1999) ACC/AHA guidelines for coronary angiography. A report of the American College of Cardiology/ American Heart Association Task Force on practice guidelines (Committee on Coronary Angiography). Developed in collaboration with the Society for Cardiac Angiography and Interventions. J Am Coll Cardiol 33:1756-1824

16. Rizzo RJ, Aranki SF, Aklog L, Couper GS, Adams DH, Collins JJ Jr et al (1994) Rapid noninvasive diagnosis and surgical repair of acute ascending aortic dissection. Improved survival with less angiography. J Thorac Cardiovasc Surg 108:567-574

17. Achenbach S, Anders K, Kalender WA (2008) Dual-source cardiac computed tomography: image quality and dose considerations. Eur Radiol 18:1188-1198

18. Vanhoenacker PK, Heijenbrok-Kal MH, Van Heste R, Decramer I, Van Hoe LR, Wijns W et al (2007) Diagnostic performance of multidetector CT angiography for assessment of coronary artery disease: meta-analysis. Radiology 244:419-428

19. Sun Z, Jiang W (2006) Diagnostic value of multislice computed tomography angiography in coronary artery disease: a metaanalysis. Eur J Radiol 60:279-286

20. Frauenfelder T, Appenzeller P, Karlo C, Scheffel H, Desbiolles L, Stolzmann P et al (2008) Triple rule-out CT in the emergency department: protocols and spectrum of imaging findings. Eur Radiol. 19:789-799

21. Schertler T, Scheffel H, Frauenfelder T, Desbiolles L, Leschka S, Stolzmann P et al (2007) Dual-source computed tomography in patients with acute chest pain: feasibility and image quality. Eur Radiol 17:3179-3188

22. Stolzmann P, Leschka S, Scheffel H, Krauss T, Desbiolles L, Plass A et al (2008) Dual-source CT in step-and-shoot mode: noninvasive coronary angiography with low radiation dose. Radiology 249:71-80 